\title{
PLANETARY AND PROTO-PLANETARY NEBULAE IN THE IRAS TWO-COLOUR DIAGRAM
}

\author{
P. GARCíA-LARIO \\ LAEFF - Estación de Villafranca del Castillo. Apdo. 50727. E-28080 Madrid (Spain) \\ A. MANCHADO \\ Instituto de Astrofísica de Canarias. E-38200, La Laguna, Tenerife (Spain) \\ and \\ S.R. POTTASCH \\ Kapteyn Laboratorium, Postbus 800. NL-9700, AV Groningen (The Netherlands)
}

\begin{abstract}
We present a study of the distribution of post-AGB stars and planetary nebulae (PNe) in the IRAS two-colour diagram. From the analysis of the distribution of spectral types of post-AGB stars in this diagram we conclude that the position at which a star leaves the infrared sequence of colours observed in AGB stars depends only on the initial mass of the progenitor star. The evolution after the end of the AGB phase takes place, in a first approximation, at a constant value of $[25]-[60]$.

- Low mass stars leave this "infrared main sequence" (IRMS) at a low value of [25] - [60], evolving slowly to the right in the IRAS two-colour diagram towards hotter spectral types. When the light of the central star is seen again after the phase of total obscuration, the spectral type is still $\mathrm{K}$ or $\mathrm{M}$.

- High mass stars leave the IRMS at a higher value of [25]- [60], evolving faster towards hotter spectral types. When the central star is seen again it shows an $\mathrm{A}$ or B spectral type.

The position of the RV-Tauri stars in the IRAS two-colour diagram is consistent with their possible nature as low mass post-AGB stars, while many of the so called "BQ[ ] stars" are probably high mass progenitors of type I PNe. The presence of hot dust, detected in the near infrared, and $\mathrm{H} \alpha$, sometimes with strong P-Cygni profiles, in many of these post-AGB stars confirms that the mass loss does not completely stop after the end of the AGB phase.

Similar results are found when we study the distribution of evolved planetary nebulae in this diagram. From the study of the galactic distribution, excitation class and type of $\mathrm{PNe}$ we conclude that low mass PNe are located in the lower part of the IRAS two-colour diagram while high mass PNe can only be found in the upper part of it. We suggest that bipolarity in PNe is a phenomenon that could be related to the post-AGB activity in high mass stars. The results here presented are consistent with the observational results found in $\mathrm{OH} / \mathrm{IR}$ stars and confirms the new evolutionary interpretation of the sequence of infrared colours observed by IRAS.
\end{abstract}

